\title{
Paper Simulations of the MAC Layer in the LoRaWAN Networks
}

\author{
Maciej Piechowiak ${ }^{1}$ and Piotr Zwierzykowski ${ }^{2}$ \\ ${ }^{1}$ Kazimierz Wielki University, Bydgoszcz, Poland \\ ${ }^{2}$ Faculty of Computing and Telecommunications, Poznan University of Technology, Poznan, Poland
}

https://doi.org/10.26636/jtit.2020.144120

\begin{abstract}
The Internet of Things is changing the approach to data transmission, protocol design and network services. The challenge faced by designers of IoT solutions is to determine the scalability of a given technology, with a particular emphasis placed on unlicensed frequency bandwidth (ISM) transmission in highly urbanized areas. Because the design and implementation of a wireless network for the Internet of Things, relying on each of the presented technologies, is expensive and time consuming, it must be preceded by a performance assessment based on computer simulations. The literature contains various approaches to modeling the mechanisms of the MAC layer of LoRa technology and to its implementation in LoRaWAN networks. The article provides an overview of major LoRa MAC network simulators. It presents and comments on the most important research results obtained by the authors of the aforementioned software.
\end{abstract}

Keywords-Internet of Things, LoRa, LoRaWAN, LPWAN, simulations.

\section{Introduction}

The fundamental requirements applicable to Internet of Things (IoT) data transmission technologies include low power consumption, low cost and low complexity of end nodes capable of transmitting over long distances. With such assumptions kept in mind, the end devices may be battery operated or may operate autonomously, with the support of energy harvesting. The communication range covers distances from hundreds of meters to several kilometers, and the network is based on the star topology. Thus, routing-related problems in this type of network are not significant and are not considered here.

Among the solutions dedicated to the IoT, three competing categories are mentioned, differing in range, cost and throughput, that will dominate these networks. They include the following:

- Low rate wireless personal area network (LRWPAN) technologies that are relied upon to create short range networks interconnecting devices owned by an individual or constituting a building network. These networks provide low data rates and short range communications in order to focus on low energy consumption and saving battery capacity. As far as channel access is concerned, IEEE 802.15.4 uses the carrier sense multiple access with collision avoidance (CSMA/CA) technology. This multiplexing approach allows multiple nodes access the same channel at different times, without any interference. Most transmissions consist of short packets and occur with a very low duty cycle (less than $1 \%$ ), minimizing power consumption. The defined minimum TX power level is $-3 \mathrm{dBm}$ or $0.5 \mathrm{~mW}$. Most modules use $0 \mathrm{dBm}(1 \mathrm{~mW})$.

- NB-IoT and LTE-M standards operate in licensed bands and leverage the already existing cellular network coverage to provide access to IoT nodes. New standards will play a key role in the development of $5 \mathrm{G}$ networks which serve as a foundation stone for mass-scale implementation of IoT solutions [1].

- Low power wide area networks (LPWAN) which provide wireless connections using the star topology and long distance transmission in the unlicensed frequency bands below $1 \mathrm{GHz}$. Sigfox uses the ultra narrow band (UNB) radio technology at $868 \mathrm{MHz}$ in Europe and in the Middle East, and $902 \mathrm{MHz}$ in North America. Thanks to its bandwidth of 192 $\mathrm{kHz}$, it allows to transmit more signals simultaneously. It is possible to communicate over long distances while displaying high noise immunity levels. The potential of losing a packet is minimized by sending three copies of the data packet at random frequencies. A Sigfox frame carries 12 bytes of data, and together with the data needed to control the transmission, it is no more than 26 bytes in size. The LoRaWAN technology, in turn, is becoming a leader of LPWAN range of solutions due to ultra-low power consumption, operation in the unlicensed band (similarly to Sigfox) and exploits a new spread spectrum physical layer design that enables higher receiver sensitivity and better coverage. The past three years have seen the research community placing an increasing emphasis on LoRaWAN. Based on data presented at The Things Conference, the LoRaWAN IoT ecosystem is the largest, with over 500 companies and over 3,000 members deploying that particular solution.

It turns out that the LoRaWAN technology enjoys - due to its low radio emissions, long range and, most importantly, 
wide support from the global community - the greatest popularity among both IoT enthusiasts and scientific communities [2].

The design and implementation of a wireless network for IoT, relying on each of the aforementioned technologies, is expensive and time consuming. Therefore, it must be preceded by a performance assessment using computer simulations. Mathematical models are required to assess the impact of specific network parameters, to assess the performance of the solution and, consequently, its usability.

The article is divided into five sections. Section 1 discusses the IoT solutions, while Section 2 describes basics of the LoRaWAN technology. Section 3 covers performance and restrictions of the MAC layer. Section 4 reviews application of the LoRaWAN network simulation at the MAC layer, and presents the simulation results. Section 5 summarizes the work performed and presents the conclusions drawn.

\section{LoRaWAN Technology Overview}

LoRaWAN is a network standard proposed by the LoRa Alliance [3], using proprietary LoRa modulation (based on the chirp spread spectrum modulation technique) - developed and owned by Semtech Company [4]. The main modulation parameter is the spreading factor (SF) that affects the data rate and the range of radio transmission (the value of this parameter varies from 7 to 12). Signals modulated with different SF parameters are orthogonal, so they may be decoded when they are transmitted at the same time and on the same frequency [5]. Moreover, it is possible to decode signals using the same SF when the difference in their power levels is greater than $6 \mathrm{~dB}(4 \mathrm{~mW})$ [4].

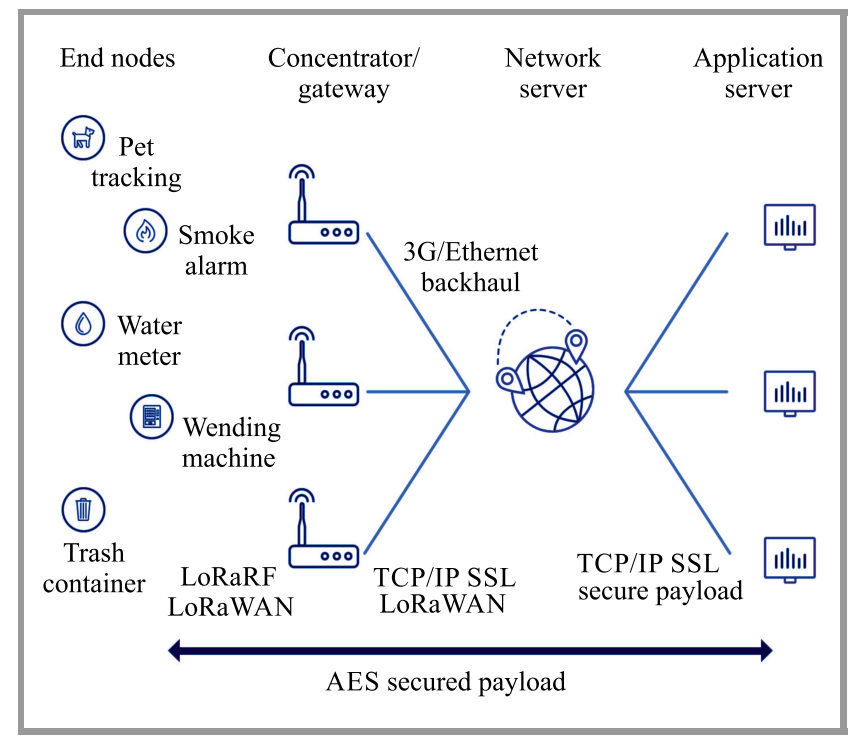

Fig. 1. Architecture of LoRaWAN system.

At the network access layer (MAC), LoRaWAN architecture defines an open protocol standardized by the LoRa
Alliance. The LoRaWAN network architecture relies on a star topology, in which the end nodes (e.g. sensors, systems measuring physical values) communicate only with a finite number of LoRaWAN gateways in a given area and do not communicate with each other (Fig. 1). Therefore, the gateways perform the function of so-called packet relays in communicating with the network server, by encapsulating raw data into IP packets using TCP or UDP protocols. The network server also allows to send downlink packets towards end nodes. The details of this transmission are based on the class of the end device - the standard defines three classes of end devices: A, B and C.

In order to save energy, Class A devices remain in the sleep mode for most of the time, opening two reception windows (RX) only 1 and 2 seconds after the end of the packet transmission (from the end device to the gateway). This mechanism allows the end devices to send acknowledgment packets (the ACK message is sent in one of the two windows after the network server has received the message correctly). For transmitting in the first window, the same frequency channel is used. The transmission relying on the second window takes place via the $869.525 \mathrm{MHz}$ channel, with a spreading factor of 12 and transmission power increased to $24 \mathrm{dBm}(250 \mathrm{~mW})$.

To increase the system's transmission capabilities toward the end nodes, Class B devices open reception windows at specific, scheduled times. The gateway transmits a beacon downlink to the Class B end devices in order to synchronize and let the web server know when a specific end device will listen for downlink traffic. Class $\mathrm{C}$ devices open reception windows continuously, being available for downlink traffic all the time (except for the time during which they are transmitting).

The LoRaWAN protocol relies on mechanisms that ensure reliable and secure communication. The adaptive data rate (ADR) mechanism allows to dynamically manage link parameters to increase the packet delivery rate. Transmission parameters may be managed at both end device and network server sides. According to the standard's documentation [6], the end device first tries to tune connectivity increasing its transmit power. If this turns out to be insufficient, it will continue to decrease the data rate.

\section{LoRa MAC Performance}

LoRa uses the ALOHA technique to access the transmission channel. This is one of the simplest and least effective access methods. In a pure ALOHA, the probability of collision is proportional to the number of users of the common transmission channel, but LoRa is characterized by a variable packet length - this increases the collision rate [7]. Therefore, many manufacturers implement the listen-before-talk (LBT) mechanism in addition to the standard solution. LBT means that the end nodes enter the receiving mode and check the interference signal level before starting the transmission (Murata modem firmware enables LBT while selecting Japan and Korean LoRa frequency 
ranges) [8]. The impact of the LBT mechanism is also the subject of simulation studies [9].

The important limitations affecting scalability of LoRaWAN networks include interference caused by other wireless technologies operating in the same ISM band, and self-interference. In Europe, LoRaWAN operates on $868 \mathrm{MHz}$ band channels. Similar technologies dedicated to the IoT, such as Sigfox, IEEE 802.15.4g or Z-Wave, also operate in this band. Therefore, they affect the MAC layer of LoRa. Such cross-technology interference (CTI) is the subject of research as well [10], [11].

LoRa transmission at the MAC layer level is also exposed to interference from end nodes and downlink transmission from LoRaWAN gateways, caused by:

- packet collisions that occurs on the same frequency channel and due to the same spreading factor SF,

- collisions of packets sent downlink to the end device in the first transmission window (RX1) and packets sent to the gateway by other devices on the same channel,

- half-duplex communication between the LoRaWAN gateway and the end node (collision between the message transmitted from the end node during the transmission of the message from the gateway to the node) when the gateway is transmitting data or is just starting the transmission,

- unavailability of RX1 and RX2 reception windows due to other transmissions or duty cycle restrictions.

In addition, the following standard-related restrictions should be taken into account:

- ETSI regulations for the 863-870 MHz ISM band define the limits of the maximum duty cycle value at $0.1 \%$ or $1 \%$, depending on the selected sub-band, which limits the bandwidth of devices and the total network capacity - the number of devices in a given area may be large, but they are limited to a few bytes of data per day,

- in computer modeling, in order to determine the radio range of wireless network nodes, it is important to choose the propagation model and to select radio transmission parameters; it was shown in [12], [13] that the packet loss rate does not exceed $10 \%$ when the range is less than $2 \mathrm{~km}$ and when SF is 9-12, and that it increases to above $60 \%$ when the range exceeds $3.4 \mathrm{~km}$ and $\mathrm{SF}$ is 12.

\section{Network Models and Simulators}

Network performance is difficult to evaluate based on measurements performed in real networks. The number of parameters and potential scenarios precludes the construction of a real-world, large network. Therefore, models of self-interference, as well as of techniques used for accessing the common transmission channel and for controlling data transmission (e.g. ACK, JOIN) enable researchers to evaluate the performance of LoRa- and LoRaWAN-based operations.

A review of the literature shows that most articles presenting LoRa network simulations analyze access to a common channel only for traffic generated by the end nodes. In [7], the authors included specific assumptions of a model simulating the MAC sublayer of a LoRa network in the LoRaSim application, written in Python, using the SimPy framework. However, some simplifications were made, e.g. the effect of imperfect orthogonality of messages generated in the same channel with different SF was omitted. The main metric used in the simulation to evaluate the quality of the system is called data extraction rate (DER), and is determined as the ratio between the amount of received messages and transmitted messages over a specific period of time.

Figure 2 (presented in [7]) shows the dependence of the abovementioned parameter on three sets of transmitting node parameters for different SF values. For the SN3 set, all nodes share the same SF. For $S N^{4}$, the parameters are selected to minimize the airtime at a constant transmission power of $14 \mathrm{dBm}(25 \mathrm{~mW})$, while for $S N^{5}$ airtime is minimized, followed by a transmission power that still allows the end node to successfully reach the gateway. Based on this configuration, it may be observed that the DER parameter drops below $50 \%$ for a gateway serving more than 900 devices. In turn, assuming that the ratio of correctly received packets (DER) equals $>90 \%$, the number of serviceable end nodes is 1600 .

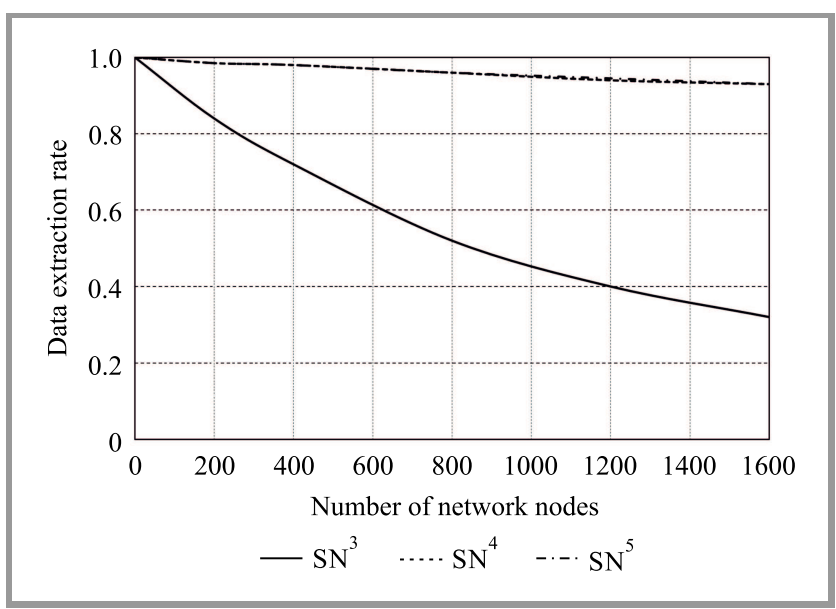

Fig. 2. Data extraction rate (DER) versus number of nodes for three transmitter parameters sets [7].

LoRaWANSim [14] extends the LoRaSim simulator by supporting MAC layer mechanisms of the LoRaWAN protocol which supports bidirectional communication. It implements a more complicated LoRaWAN MAC protocol instead of the features of the ALOHA protocol used in the LoRaSim simulator. Downlink transmission to the end node is crucial in IoT applications due to handshakes, acknowledgement traffic (i.e. ACK, LinkCheck, Duty Cycle, 
RXParam-Setup), and key exchange in cryptographic algorithms, and has been taken into account.

LoRaWANSim provides a $1 \%$ duty cycle for most European sub-bands. It also implements a realistic collision model in which collisions for uplink and downlink traffic do not occur even when the transmission is carried out at the same time and on the same channel and the same SF. The end device will only receive the packet sent by the gateway, even if it experiences a signal with a higher received signal strength indicator (RSSI) from its neighboring nodes, if it is part of a group of end nodes far away from the gateway. This simulator considers the retransmission strategy as well. Retransmission occurs when the reception of a packet is not confirmed (e.g. due to a collision or duty cycle limitations). In that case, the LoRaWAN specification recommends to transmit it up to 8 times. If 8 consecutive attempts fail, the application should be notified. Moreover, the specification recommends that the end nodes limit the transmission data rate every two unsuccessful transmission attempts, in order to increase the robustness of uplink connectivity.

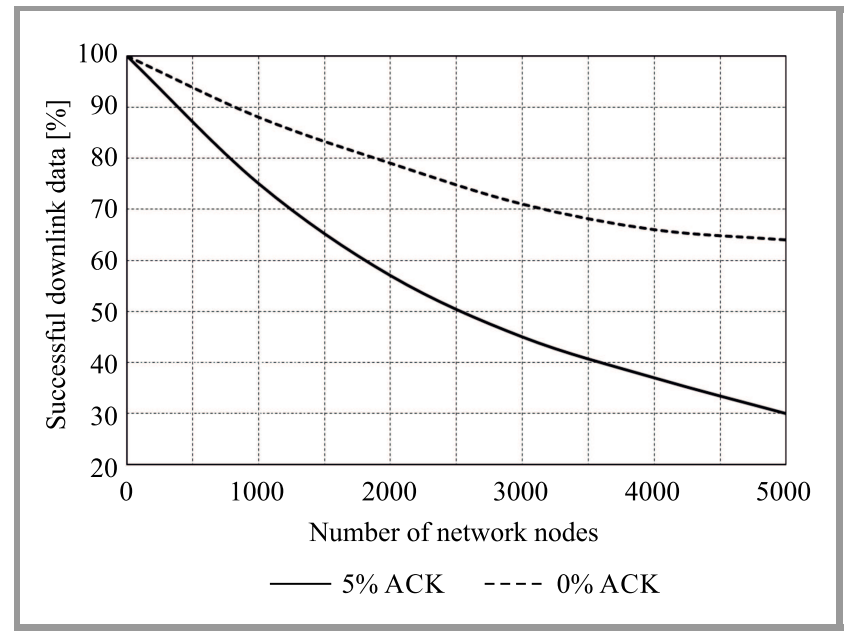

Fig. 3. Impact of network size on successful downlink data transmission [14].

The main conclusion of the research conducted in [14] is an unreliable downlink transmission caused by gateway duty cycle limitations and collisions. Figure 3 (presented in [14]) shows the percentage of successful downstream transmissions with and without confirmation (ACK). These conclusions may be of particular importance when considering the implementation of LoRaWAN protocols in upper layers that require two-way communications (e.g. IPv6 stack).

Data rate reduction experienced when no further ACK messages have been received is an important problem that surfaces in this study. Such a strategy is correct when losses at higher data rates are associated with poor link quality and are not the result of exhaustion of the duty cycle limit at the gateway. Otherwise, reduction in the data rate is unreasonable, because by increasing the air time of the ACK message, the probability of collision is increased as well. Therefore, the recommended number of retransmission attempts in the LoRaWAN specification may not be appro- priate for all scenarios and largely depends on the network size. For small networks with less than 600 nodes, $90 \%$ of messages are confirmed immediately, while for networks of up to 1000 nodes, $95 \%$ of messages are confirmed after two retransmissions.

The increase in energy consumption is another important phenomenon associated with retransmissions. Figure 4 (presented in [14]) shows the increase in battery drainage for data transmission with retransmissions, for networks with 1000 and 5000 nodes, respectively.

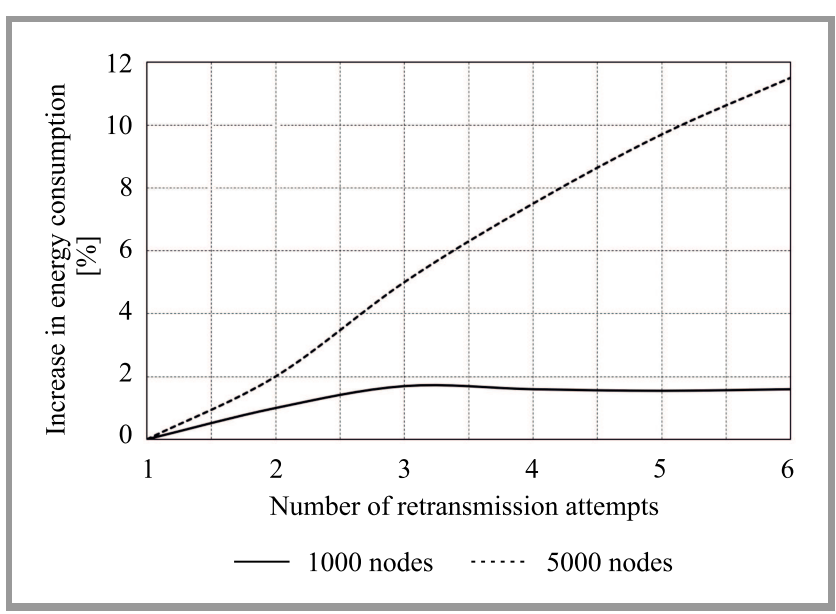

Fig. 4. Energy consumption versus the number of transmission attempts [14].

Indicating the high collision rate and the adverse impact of retransmission in the simulators presented earlier, the LoRaFREE simulator which uses synchronized bulk data transmission was proposed [15]. It also uses Simpy and the long-distance path loss model of LoRaSim [7]. This solution assumes that the data is buffered before transmission and sent in scheduled time slots. It includes, in LoRaWAN packets sent toward the end devices, basic transmission parameters, such as frequency channels, transmitter powers, spreading factors and time slots. In addition, longer packages were used, which reduced the overhead of MAC headers. Hence, scalability problems were overcome - the number of collisions and group notifications was minimized. The authors showed that, due to such an approach, the operating time of the battery-powered devices was increased more than five-fold, with the data delivery ratio exceeding $99 \%$.

The data collection process takes place periodically at intervals that are known to the gateway and end devices. In order to schedule each data collection period, the gateway must know the number of end devices that store the data to be sent, the amount of buffered data, and estimated path loss. Then, the gateway broadcasts the schedule and synchronizes all end devices with the same time reference.

In LoRaFREE, retransmissions are scheduled in such a way that devices with the same spreading factor transmit sequentially, and devices with different spreading factors transmit simultaneously. The transmission was organized into six 
parallel frames that correspond to six SFs (7-12). Each frame consists of a specific number of uplink slots and one downlink slot at the end of the frame. The end nodes send join-request messages to the gateway, containing the size of the data to be sent and the RSSI parameter of the message. Based on this data, the gateway determines the SF that the device should use in the scheduled transmission that takes place in the slot of the above-mentioned frame.

Due to the fact that transmissions with different SFs are not completely orthogonal and cause mutual interferences, it is necessary to determine the threshold difference between the strength of the reference transmission and the interfering transmissions at which both signals may be correctly received. For this purpose, the results of experimental studies presented in [5] were used, and the interference thresholds among the spreading factors are presented in Table 1.

Table 1

Interference thresholds $[\mathrm{dBm}]$

\begin{tabular}{|c|c|c|c|c|c|c|}
\hline \multirow{2}{*}{$S F_{\text {ref }}$} & \multicolumn{7}{|c|}{$S F_{\text {int }}$} \\
\cline { 2 - 7 } & 7 & 8 & 9 & 10 & 11 & 12 \\
\hline 7 & 1 & -8 & -9 & -9 & -9 & -9 \\
8 & -11 & 1 & -11 & -12 & -13 & -13 \\
9 & -15 & -13 & 1 & -13 & -14 & -15 \\
10 & -19 & -18 & -17 & 1 & -17 & -18 \\
11 & -22 & -22 & -21 & -20 & 1 & -20 \\
12 & -25 & -25 & -25 & -24 & -23 & 1 \\
\hline
\end{tabular}

The authors consider two scenarios for scheduling data transmissions in the proposed simulator (when the algorithm allocates the spreading factors so as to minimize data collection time and when the energy minimization objective is considered) and compare the results obtained with two other LoRaWAN implementations, named Legacy LoRaWAN and Delayed LoRaWAN, respectively. The Legacy LoRaWAN uses the standard LoRaWAN MAC, where devices comply with the Class A specification (ALOHA-type

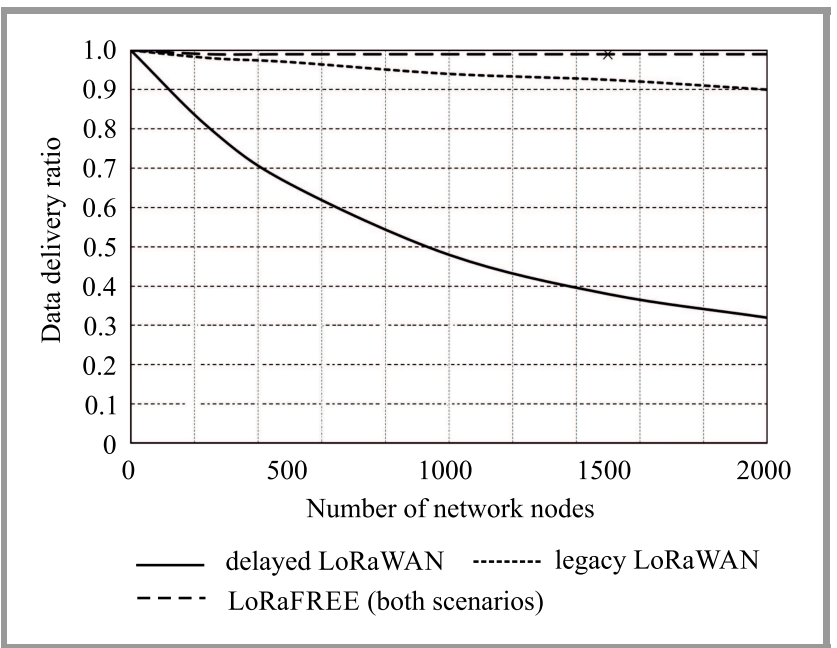

Fig. 5. Impact of network size on data delivery ratio [15].
MAC, two reception windows after each uplink and immediate transmission of the data). In the Delayed LoRaWAN, end devices buffer the data and send it in bulk, at scheduled times. The devices also comply with Class A specification and do not perform any synchronization before the transmission. A time offset is only introduced to synchronize the devices in order to minimize collisions. The end devices send long packets to reduce the overhead of MAC headers. Then, traffic was simulated where the transmissions to the gateway are not acknowledged. The application generates 20-byte packets with a Poisson distribution at the rate of 5 minutes.

Figure 5 (taken from [15]) shows that achievement of low power consumption and reduction in the duration of the transmission takes place at the expense of the data delivery ratio (DDR) which decreases significantly along with the increase in the number of network nodes for the Delay LoRaWAN model (DDR represents the ratio between correctly received packets and the initial buffer sizes of all end devices). It would seem that the pure ALOHA mechanism (Legacy LoRaWAN) generates more collisions and a higher packet loss, but the large packet size in the Delayed LoRaWAN model results in lower DDR.

\section{Conclusions and Future Works}

A literature review and research using existing simulators shows that it is possible to model the majority of MAC layer mechanisms in a LoRaWAN network using free programming environments. The simulators presented in this paper introduce further extensions of the MAC sublayer compared to the basic implementation [7]. They are written in Python and their source codes have been published. Therefore, there is no need to create complex real-world networks and advanced testbeds. Research conducted using these tools provides answers to questions concerning the error rate and network scalability limits. New mechanisms that will be implemented in subsequent versions of the specification (e.g. firmware update over the air, FUOTA) [16] basically increase the number of downlink packets and degrade the quality of transmission even further. The firmware update over the air is not part of the MAC layer but is performed at the application layer.

From the point of view of the development of new public and private LoRaWAN networks, restrictions on the maximum number of nodes and collision limitation mechanisms are important. These problems are modeled by the simulators described in this article. However, LoRaWAN network topology modeling methods are equally important. Algorithms supporting the placement of gateways in combination with radio range modeling in urban environments are main challenges faced by computer simulation environments.

The authors' participation in the research agenda aimed at sending data from electricity meters will allow to compare the simulation test outcomes with results obtained in real measurement networks using LoRaWAN technology. 


\section{References}

[1] "NB-IoT and LTE-M in the 5G context", GSMA report, May 2018 [Online]. Available: https://www.gsma.com/iot/wp-content/ uploads/2018/05/GSMA-5G-Mobile-IoT.pdf

[2] K. Mekki, E. Bajic, F. Chaxel, and F. Meyerb, "A comparative study of LPWAN technologies for large-scale IoT deployment", ICT Express, vol. 5, no. 1, pp. 1-7, 2019 (DOI: 10.1016/j.icte.2017.12.005).

[3] "LoRa Alliance" [Online]. Available: https://www.lora-alliance.org

[4] "Semtech Corporation" [Online]. Available: http://semtech.com

[5] D. Croce, M. Gucciardo, S. Mangione, G. Santaromita, and I. Tinnirello, "Impact of LoRa imperfect orthogonality: Analysis of link-level performance", IEEE Commun. Lett., vol. 22, no. 4, pp. 796-799, 2018 (DOI: 10.1109/LCOMM.2018.279705).

[6] N. Sornin and A. Yegin, "LoRaWAN 1.1 Specification", Oct. 2017 [Online]. Available: https://lora-alliance.org/sites/default/files/ 2018-04/lorawantm_specification_-v1.1.pdf

[7] M. C. Bor, U. Roedig, T. Voigt, and J. M. Alonso, "Do LoRa Low-Power Wide-Area Networks Scale?", in Proc. of the 19th ACM Int. Conf. on Model., Anal. and Simul. of Wirel. and Mob. Syst MSWiM'16, Malta, 2016, pp. 59-67 (DOI: 10.1145/2988287.2989163).

[8] "LoRa Module FAQ", Murata Electronics, 2020 [Online]. Available: https://www.murata.com/en-eu/support/faqs/products/ lpwa/lora/software/0006

[9] T. To and A. Duda, "Simulation of LoRa in NS-3: Improving LoRa performance with CSMA", in Proc. IEEE Int. Conf. on Commun. ICC 2018, Kansas City, MO, USA, 2018 (DOI: $10.1109 /$ ICC.2018.8422800).

[10] C. Orfanidis, L. M. Feeney, M. Jacobsson, and P. Gunningberg, "Investigating interference between LoRa and IEEE 802.15.4g networks", in Proc. of the IEEE 13th Int. Conf. on Wirel. and Mob. Comput., Network. and Commun. WiMob 2017, Rome, Italy, 2017 (DOI: 10.1109/WiMOB.2017.8115772).

[11] J. Haxhibeqiri et al., "Sub-gigahertz inter-technology interference. How harmful is it for LoRa?, in Proc. of the IEEE Int. Smart Cities Conf. ISC2 2018, Kansas City, MO, USA, 2018 (DOI: 10.1109/ISC2.2018.8656742).

[12] O. Georgiou and U. Raza, "Low power wide area network analysis: Can LoRa scale?", IEEE Wireless Commun. Lett., vol. 6, no. 2, pp. 162-165, 2017 (DOI: 10.1109/LWC.2016.2647247).

[13] A. Augustin, J. Yi, T. H. Clausen, and W. M. Townsley, "A study of LoRa: long range \& low power networks for the Internet of Things", Sensors, vol. 16, no. 9, 2016 (DOI: 10.3390/s16091466).

[14] A. Pop, U. Raza, P. Kulkarni, and M. Sooriyabandara, "Does bidirectional traffic do more harm than good in LoRaWAN based LPWA networks?", in Proc. IEEE Global Communi. Conf. GLOBECOM 2017, Singapore, 2017 (DOI: 10.1109/GLOCOM.2017.8254509).

[15] K. Q. Abdelfadeel, D. Zorbas, V. Cionca, and D. Pesch, "FREE-Finegrained scheduling for reliable and energy efficient data collection in LoRaWAN", IEEE Internet of Things J., vol. 7, no. 1, pp. 669-683, 2019 (DOI: 10.1109/JIOT.2019.2949918).

[16] "FUOTA Process Summary Technical Recommendation", LoRa Alliance, Jan. 2019 [Online]. Available: https://lora-alliance.org/sites/ default/files/2019-04/tr002-fuota_process_summary-v1.0.0.pdf



Maciej Piechowiak received his M.Sc. degree from the University of Technology and Life Sciences, Bydgoszcz, Poland, in 2002, and his Ph.D. degree from the Poznan University of Technology, Poznan, Poland, in 2010. He is currently an Assistant Professor at the Institute of Computer Science of the Kazimierz Wielki University, Bydgoszcz, Poland. Dr. Maciej Piechowiak is an author and co-author of dozens of articles published in journals and conference proceedings (several conference awards). He has served as a Guest Editor and an Editorial Board member of two international journals and as TPC member of several international conferences.

(iD) https://orcid.org/0000-0001-8346-5812

E-mail: mpiech@ukw.edu.pl

Uniwersytet Kazimierza Wielkiego

Instytut Informatyki

Kopernika 1

85-074 Bydgoszcz, Poland



Piotr Zwierzykowski received his Ph.D. and D.Sc. degrees in Telecommunications from the Poznan University of Technology, Poland, in 2002 and 2015, respectively. Since 1995, he has been working at the Faculty of Computing and Telecommunications, Poznan University of Technology. Currently, he works as a professor at the Institute of Communication and Computer Networks. Piotr Zwierzykowski is the author or co-author of over 200 papers and four books. He is engaged in conducting research and teaching activities in the area of computer networks, multicast routing algorithms and protocols, as well as performance analysis of multiservice switching systems.

(iD) https://orcid.org/0000-0001-5609-1026

E-mail: piotr.zwierzykowski@put.poznan.pl Institute of Communication and Computer Networks Faculty of Computing and Telecommunications

Poznan University of Technology

Pl. Marii Skłodowskiej-Curie 5

60-965 Poznan, Poland 